\title{
Fabricación y caracterización de carbón activado y de nanoplaquetas de carbón a partir de Guadua angustifolia Kunth para aplicaciones en electrónica
}

\author{
Jhon Jairo Prías-Barragán ${ }^{1,2, *}$, Narly Andrea Echeverry-Montoya ${ }^{1}$, Hernando Ariza-Calderón ${ }^{1}$ \\ ${ }^{1}$ Laboratorio de Optoelectrónica, Universidad del Quindío, Armenia, Colombia \\ ${ }^{2}$ Programa de Tecnología en Instrumentación Electrónica, Universidad del Quindío, Armenia, Colombia
}

\begin{abstract}
Resumen
Se fabricaron y caracterizaron muestras de carbón activado y nanoplaquetas de carbón obtenidas de Guadua angustifolia Kunth empleada como precursor para aplicaciones en electrónica flexible. El carbón activado se obtuvo en un sistema de pirólisis bajo atmósfera controlada de nitrógeno a una temperatura de $573 \mathrm{~K}$ durante una hora y las nanoplaquetas, a una temperatura de $973 \mathrm{~K}$ durante una hora. El carbón se activó empleando hidróxido de sodio e hidróxido de potasio con una temperatura de activación de $973 \mathrm{~K}$. Las nanoplaquetas se obtuvieron mediante procesos de molienda mecánica en mortero y procesos de cavitación durante seis horas. Las muestras de carbón activado se caracterizaron mediante isotermas de adsorción y se encontró un área superficial de 408,0 $\mathrm{m}^{2} / \mathrm{g}$ y $308,9 \mathrm{~m}^{2} / \mathrm{g}$ para el carbón activado con hidróxido de sodio e hidróxido de potasio, respectivamente. Se utilizó la difracción de rayos X para determinar la presencia de electrólitos remanentes del proceso de activación. Las imágenes obtenidas con el microscopio electrónico de barrido revelaron la estructura porosa del carbón y la presencia de las sales electrolíticas remanentes. Mediante voltametría cíclica se determinó una capacitancia específica máxima de $111 \mathrm{~F} / \mathrm{g}$. El carbón activado se empleó en la fabricación de un supercondensador flexible y se logró una capacitancia de 7,9 mF. Las nanoplaquetas se caracterizaron mediante las técnicas de difracción de rayos $\mathrm{X}$, microscopía electrónica de barrido, espectrometría infrarroja con transformada de Fourier y microscopía electrónica de transmisión, con las que se corroboró la presencia de nanoplaquetas de grafito oxidado con espesores inferiores a $13 \mathrm{~nm}$; las curvas de intensidad-voltaje evidenciaron un comportamiento no lineal, atribuido a efectos de percolación de los portadores de carga eléctrica. Estos resultados sugieren que el carbón activado y las nanoplaquetas de carbón son excelentes candidatos para aplicaciones electrónicas.
\end{abstract}

Palabras clave: carbón activado, Guadua angustifolia Kunth, nanoplaquetas, electrónica flexible.

Fabrication and characterization of activated carbon and carbon nanoplatelets from Guadua angustifolia Kunth for their application in electronics

\begin{abstract}
We report the fabrication and characterization of activated carbon and carbon nanoplatelets obtained from Guadua angustifolia Kunth for their application in flexible electronics. The activated carbon samples were obtained in a controlled pyrolysis system under nitrogen atmosphere at a temperature of $573 \mathrm{~K}$ for one hour, and the nanoplatelets at $973 \mathrm{~K}$ for one hour. The charcoal was activated using potassium hydroxide and sodium hydroxide at an activation temperature of $973 \mathrm{~K}$. The nanoplatelets samples were obtained by mechanical grinding in a mortar, and cavitation for six hours. The activated carbon samples were characterized by adsorption isotherms, and we found a surface area of $408.0 \mathrm{~m}^{2} / \mathrm{g}$ and $308.9 \mathrm{~m}^{2} / \mathrm{g}$ for the carbon activated with sodium hydroxide and potassium hydroxide, respectively. $\mathrm{X}$-ray diffraction was performed and the presence of electrolytes remaining from the activation process was determined. Scanning electronic microscopy images showed the porous carbon structure and allowed to identify the presence of the remaining electrolyte salts. Cyclic voltammetry was performed and a maximum specific capacitance of $111 \mathrm{~F} / \mathrm{g}$ was determined. The activated carbon was used in the manufacture of a flexible supercapacitor, achieving a capacitance of $7.9 \mathrm{mF}$. The nanoplatelets were characterized by X-ray diffraction, scanning electronic microscopy, transmission electron microscopy and Fourier transform infrared spectroscopy techniques, corroborating the presence of oxidized graphite nanoplatelets with thicknesses below $13 \mathrm{~nm}$; using current-voltage curves we found a nonlinear behavior attributed to the percolation effects of the electric charge carriers. These results suggest that activated carbon and carbon nanoplatelets samples are excellent candidates for electronic applications.
\end{abstract}

Key words: Activated carbon, Guadua angustifolia Kunth, nanoplatelets, flexible electronics. 


\section{Introducción}

La producción de carbón depende, entre otros aspectos, de la disponibilidad, la calidad y los costos de la materia prima, parámetros que de acuerdo a las propiedades intrínsecas del precursor determinan las propiedades físicas y químicas del carbón resultante. El carbón tiene múltiples aplicaciones en diversos campos como la medicina, el tratamiento de aguas, la eliminación de olores y sabores, como agente decolorante en la industria del azúcar, así como para uso en máscaras de adsorción de gases tóxicos, entre otras (PríasBarragán, et al., 2011). La guadua como precursor en la producción de carbón se ha estudiado poco, a pesar de que su cultivo tiene una alta tasa de producción y de reposición y un bajo costo de mantenimiento, lo cual la convierte en un excelente candidato para la producción de carbón vegetal y carbón activado. El carbón activado presenta extensas áreas de superficie, tiene una gran conductividad eléctrica, es de bajo costo y tiene una elevada estabilidad química (Jayalakshmi \& Balasubramanian, 2008), por lo que es un material ampliamente utilizado en la fabricación de supercondensadores. Por otra parte, los avances en el desarrollo de nanoplaquetas de carbón a partir de carbón vegetal y el estudio de sus propiedades físicas ha propiciado la integración de estos materiales al desarrollo de sensores electrónicos y otros dispositivos avanzados (Llobet, 2013; Liu, Zhao, Zhang, 2014). Se ha encontrado que las interesantes propiedades de transporte del grafeno también pueden presentarse en las nanoplaquetas de carbón y de grafito y que, por lo tanto, podrían emplearse en el desarrollo de aplicaciones de tecnología avanzada en electrónica (Rozploch, et al., 2007). En este contexto, se presenta aquí el proceso de fabricación y caracterización de carbón activado y nanoplaquetas de carbón obtenidas a partir de Guadua angustifolia Kunth utilizada como precursor, y de su implementación en un supercondensador y en la configuración de un micropuente para aplicaciones en electrónica flexible.

\section{Materiales y métodos}

La carbonización del material precursor se hizo mediante descomposición térmica en una atmósfera controlada y con un sistema automatizado de pirólisis. Los productos resultantes de la carbonización fueron gases, líquidos y carbón, cuyas cantidades relativas dependieron de las propiedades del precursor y de los parámetros de operación del equipo de calentamiento (Lesme, 2010). Las muestras de G. angustifolia Kunth se cortaron inicialmente en tabletillas de aproximadamente $3 \mathrm{~cm} \times 3 \mathrm{~cm}$ de la parte media del culmo empleando una caladora eléctrica; luego se llevaron a un reactor cilíndrico de pirólisis en el interior de una mufla con

\footnotetext{
*Correspondencia:

Jhon Jairo Prías-Barragán, jjprias@uniquindio.edu.co

Recibido: 15 de octubre de 2014

Aceptado: 16 de septiembre de 2015
}

temperaturas controladas que pueden alcanzar $1.173 \mathrm{~K}$, y un consumo de potencia eléctrica de $4.400 \mathrm{~W}$. Se aplicó vacío en el sistema y se hizo fluir dinitrógeno $\left(\mathrm{N}_{2}\right)$ para garantizar la obtención de carbón en una atmosfera no oxidante.

Las muestras de carbón activado se obtuvieron a una temperatura de carbonización de $573 \mathrm{~K}$ durante una hora. Una vez obtenido, el carbón se molió manualmente en mortero hasta obtener un tamaño de partícula menor a 180 $\mu \mathrm{m}$, lo cual se verificó pasando el material por un tamiz RoTap Model E. Posteriormente, se impregnó el carbón con los agentes de activación de hidróxido de sodio $(\mathrm{NaOH})$ e hidróxido de potasio $(\mathrm{KOH})$ en una relación agente de activación:carbón de 4:1; la muestra de carbón se sometió luego a un tratamiento térmico de impregnación a $423 \mathrm{~K}$ de temperatura durante cinco horas, la cual se aumentó después a $973 \mathrm{~K}$ durante una hora para lograr la activación. Las curvas de temperatura se establecieron a una razón de flujo de calor de $0,3 \mathrm{~K} / \mathrm{s}$ y el proceso de enfriamiento a una de $0,1 \mathrm{~K} / \mathrm{s}$. Se procedió entonces al lavado del carbón activado con agua desionizada (pura) y a la adición de ácido clorhídrico $(\mathrm{HCl})$ hasta obtener un $\mathrm{pH}$ neutro constante. En esta etapa del proceso se formaron sales de cloruro de sodio $(\mathrm{NaCl})$ y de cloruro de potasio $(\mathrm{KCl})$ solubles en agua, la mayor parte de las cuales se retiró del carbón por medio de filtración al vacío con un embudo Gooch y papel de filtro de grado 3. El carbón activado (CA) obtenido se empleó en la construcción de un dispositivo supercondensador, para lo cual el CA- $\mathrm{NaCl}$ y el CA-KCl se impregnaron con $0,5 \mathrm{~mL}$ de ácido sulfúrico $\left(\mathrm{H}_{2} \mathrm{SO}_{4}\right)$ a 0,25 molar y se dispusieron sobre una lámina flexible de aluminio; ambos electródos se colocaron paralelamente, separados mediante papel de filtro, y todo el dispositivo se encapsuló usando cinta polimérica transparente.

Por otro lado, la fabricación de las muestras de nanoplaquetas de carbón se hizo a partir del carbón vegetal obtenido a 973 $\mathrm{K}$; las muestras se trituraron manualmente en un mortero y, posteriormente se pasaron por un tamiz vibratorio Ro-Tap model E con una malla de $20 \mu \mathrm{m}$, con lo que se obtuvo una cantidad de aproximadamente $0,5 \mathrm{~g}$. A este fino polvo de carbón se lo sometió a procesos de cavitación mediante un baño ultrasónico en un equipo BRANSON de la serie 5210 durante seis horas.

Después de este procedimiento la muestra se depositó en un recipiente totalmente seco, al cual se le adicionó gradualmente agua desionizada (pura) hasta lograr la formación de una película delgada de nanoplaquetas suspendidas en la superficie del agua, proceso que tomó alrededor de cinco minutos; la película fue retirada del medio acuoso mediante una lámina de vidrio o acetato a la cual se adherían fácilmente las nanoplaquetas, que posteriormente fueron depositadas en un vaso de precipitado con $500 \mathrm{~mL}$ de agua desionizada (pura) en una razón solido:agua de $0,1 \mathrm{~g} / \mathrm{L}$. Después, la muestra se secó en una estufa de calentamiento 
marca Lindberg Blue $\mathrm{M}$ y se obtuvo un polvo ultra fino que se caracterizó y se empleó en la elaboración de dispositivos del tipo de micropuentes electrónicos.

Las muestras de carbón activado se caracterizaron mediante isotermas de adsorción aplicando el método de Brunauer, Emmet y Teller (BET) en el equipo ASAP 2020. Estos análisis permitieron el cálculo del área superficial del carbón activado empleando la adsorción de moléculas de gas nitrógeno. En la caracterización estructural se utilizó un equipo de difracción de rayos X D8 Advance Bruker. Las imágenes de microscopía avanzada se tomaron con un microscopio electrónico de barrido (scanning electronic microscope, SEM) marca JEOL JSM 6490LV. La obtención de las imágenes se logró con voltajes que variaron entre 5 y $20 \mathrm{KV}$ en un microscopio electrónico de transmisión (transmission electron microscope, TEM) JEOL 1011 JEM para variaciones de voltaje de 20 a $80 \mathrm{KV}$. Las curvas de intensidad-voltaje (I-V) se tomaron usando una fuente de corriente de precisión KEITHLEY 6220 y un nanovoltímetro KEITHLEY 2182A.

\section{Resultados y discusión}

Las muestras de carbón activado se caracterizaron mediante isotermas de adsorción. Con base en el respectivo análisis de resultados, se logró determinar un área específica para el CA- $\mathrm{NaCl}$ de $408,0 \mathrm{~m}^{2} / \mathrm{g}$ y tamaños promedio de microporo que variaron entre 0,43 y $0,80 \mathrm{~nm}$, y de mesoporo entre 2,60 y 30,00 nm (según clasificación de la norma de la Unión Internacional de Química Pura y Aplicada, International Union of Pure and Applied Chemistry - IUPAC), lo cual es característico en carbones con una buena relación entre microporos y mesoporos. Este tipo de carbones tiene una amplia aplicación en el campo de la industria de licores, el azúcar y la electrónica como electrodos para supercondensadores (Jayalakshmi \& Balasubramanian, 2008; Gamby, et al., 2001). Mediante isotermas de adsorción de nitrógeno también se logró determinar un área específica de $308,9 \mathrm{~m}^{2} / \mathrm{g}$ para el CA-KCl y tamaños promedio de microporo de entre 0,47 y $0,60 \mathrm{~nm}$ y de mesoporo de entre 2,80 y $20,00 \mathrm{~nm}$, lo cual evidencia un aumento en el tamaño de los microporos encontrados en el $\mathrm{CA}-\mathrm{KCl} \mathrm{y}$, en consecuencia, la reducción del área superficial en este carbón activado, ya que los poros más pequeños son los que contribuyen en mayor medida al área superficial del material. En la Figura 1 y la Figura 2, se presentan los difractogramas de rayos $\mathrm{X}$ de las muestras de carbón activado, para los cuales, se observó la presencia de sales electrolíticas de $\mathrm{NaCl}$ y $\mathrm{KCl}$, y se adicionaron las tarjetas PDF 00-005-0628 en el caso del CA-NaCl y la 0411472 en el del CA-KCl. Estas sales resaltan en la estructura amorfa del carbón y su presencia podría disminuir el área de la superficie del carbón activado registrada en las isotermas de adsorción debido a la obstrucción de los microporos y los mesoporos, pero también podría contribuir en los procesos de carga y descarga como material electrolítico. Además,

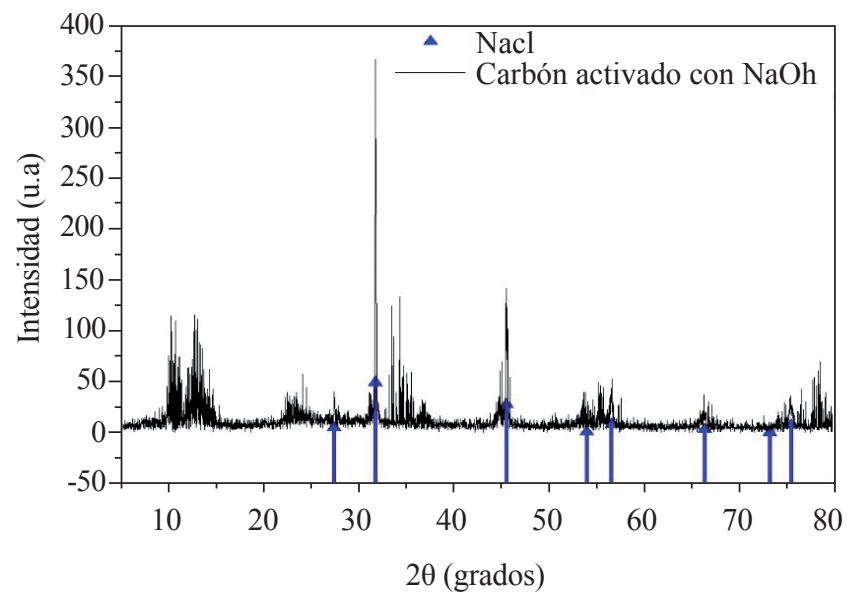

Figura 1. Difractograma de rayos $\mathrm{X}$ de $\mathrm{CA}-\mathrm{NaCl}$.

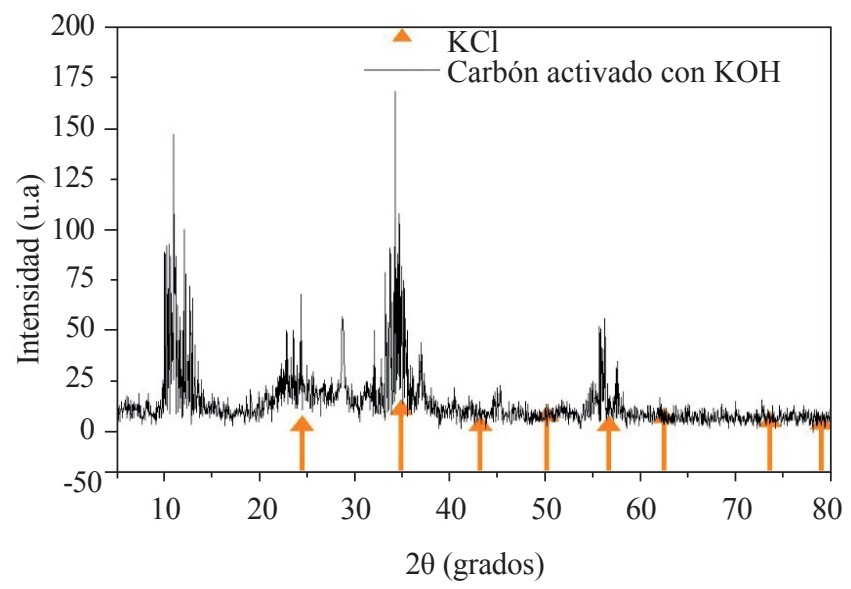

Figura 2. Difractograma de rayos $\mathrm{X}$ de $\mathrm{CA}-\mathrm{KCl}$.

el análisis cualitativo de los difractogramas sugiere que el carbón obtenido a partir del precursor G. angustifolia Kunth podría presentar una estructura del tipo del grafito oxidado, tal como ha sido propuesto por Geng, et al. (2009).

La Figura 3 presenta una micrografía obtenida con microscopio electrónico de barrido de una partícula de $\mathrm{CA}-\mathrm{NaCl}$ en la cual se puede observar la estructura porosa de la muestra y las sales remanentes de $\mathrm{NaCl}$ adheridas a la superficie del carbón. En la literatura especializada esta adherencia se ha explicado por medio del modelo de Gouy-ChapmanStern para una doble capa, de acuerdo al efecto de atracción electrostática por inducción del tipo Helmholtz (Wei \& Yushin, 2012).

En la Figura 4 se presentan las mediciones de voltametría cíclica en $\mathrm{CA}-\mathrm{NaCl}$. Con base en el respectivo análisis, se encontró el valor de la capacitancia específica $\mathrm{C}$ del electrodo de CA mediante la siguiente expresión:

$$
C=\frac{\int I d V}{v * m * \Delta V}
$$


en donde $I$ es la corriente eléctrica medida en amperios, $\Delta V$ es el potencial aplicado en voltios, $v$ es la razón de cambio del voltaje aplicado y $m$ es la masa del material activo en gramos (Guerrero-González, 2011). La capacitancia específica encontrada para el CA-NaCl a una velocidad de $10 \mathrm{mV} / \mathrm{s}$ fue de $111 \mathrm{~F} / \mathrm{g}$. Este elevado valor es muy importante para el desarrollo de dispositivos de supercondensación, pues según lo reportado por Wei \& Yushin (2012), el máximo valor de capacitancia específica medido en electrodos de CA obtenido del bambú es de $65 \mathrm{~F} / \mathrm{g}$. Este importante incremento de un orden de magnitud en el valor de la capacitancia específica se podría atribuir a las diferencias en la materia prima, ya que el bambú pertenece a una especie diferente a la de $G$. angustifolia Kunth.

En la Figura 1S, http://www.raccefyn.co/index.php/raccefyn/ article/downloadSuppFile/139/1178, se presenta el voltagrama correspondiente a CA-KCl: la capacitancia específica encontrada a una velocidad de $10 \mathrm{mV} / \mathrm{s}$ fue de $79 \mathrm{~F} / \mathrm{g}$. Los valores de capacitancia específica encontrados para CA$\mathrm{NaCl}$ y CA-KCl concordaron con los valores de $65 \mathrm{~F} / \mathrm{g}$ y 35

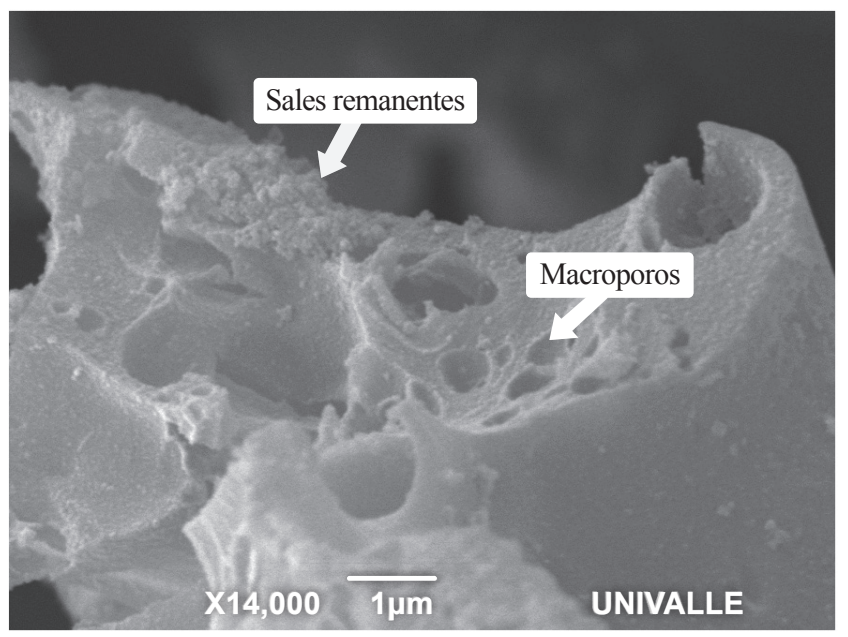

Figura 3. Micrografía de $\mathrm{CA}-\mathrm{NaCl}$ obtenida con microscopio electrónico de barrido

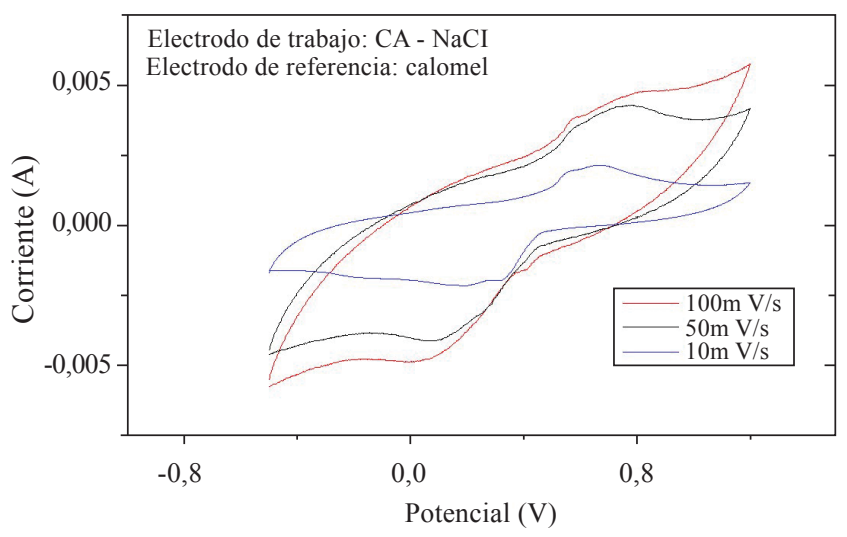

Figura 4. Voltagrama correspondiente a $\mathrm{CA}-\mathrm{NaCl}$
F/g reportados por Wei \& Yushin (2012) como los típicos de la capacitancia específica en supercondensadores con electrodos de carbón obtenidos de bambú.

Las mediciones de capacitancia en el dispositivo supercondensador se han hecho mediante el método de curvas de carga-descarga y se han obtenido valores de capacitancia de $7,9 \mathrm{mF}$ en prototipos de dispositivos de supercondensación flexibles, valor que concuerda con lo reportado por Pandolfo \& Hollenkamp (2006) en el caso de supercondensadores rígidos. Los resultados de este estudio sugieren que con el uso de electrodos de carbón activado obtenidos de la guadua se podrían incrementar de manera apreciable los valores de capacitancia en dispositivos supercondensadores flexibles gracias a la elevada capacitancia específica que exhiben los electrodos de carbón activado. Las dimensiones del dispositivo supercondensador flexible propuesto fueron de $15 \mathrm{~mm}$ de ancho, $15 \mathrm{~mm}$ de longitud y $1 \mathrm{~mm}$ de espesor. Considerando la reducida dimensión y las capacitancias exhibidas por el dispositivo en mención, se sugiere que estos dispositivos pueden ser excelentes candidatos para el desarrollo de memorias electrónicas flexibles basadas en carbón activado obtenido de la guadua.

Por otro lado, para el caso de las muestras de nanoplaquetas, en la Figura 5 se presentan imágenes de TEM en las que se puede observar la formación de placas grafíticas con alta traslucidez (espesor inferior a $13 \mathrm{~nm}$ medidos con microscopio de fuerza atómica) al paso de los electrones y con formas geométricas irregulares. Estas formas irregulares le confieren a las nanoplaquetas efectos electrostáticos importantes que propician su aglomeración, tal como se puede observar en la Figura 5 (parte izquierda), los cuales podrían influenciar de forma significativa el transporte electrónico en ellas y en dispositivos electrónicos del tipo de los micropuentes (Sangwan, et al., 2011).

En la Figura 6 se muestra la caracterización eléctrica del dispositivo en una configuración del tipo de micropuente electrónico; se puede observar el efecto no lineal en la conducción eléctrica a partir de los 2 voltios, lo que se asemeja al comportamiento eléctrico de un diodo de punto de contacto. Este comportamiento eléctrico podría atribuirse

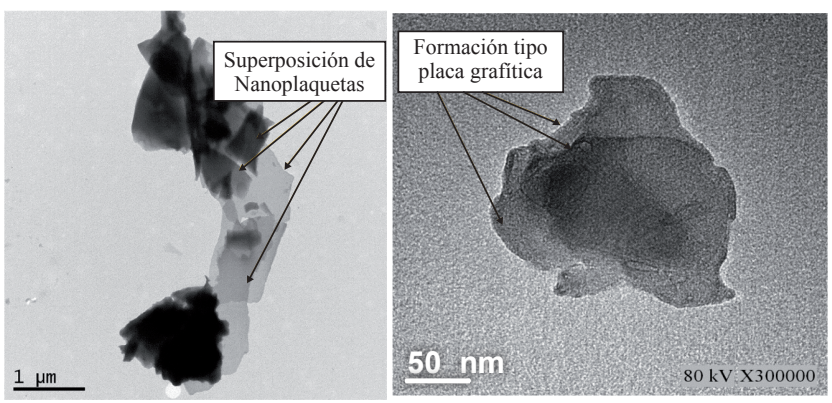

Figura 5. Imágenes de TEM en nanoplaquetas obtenidas de la guadua utilizada como precursor 


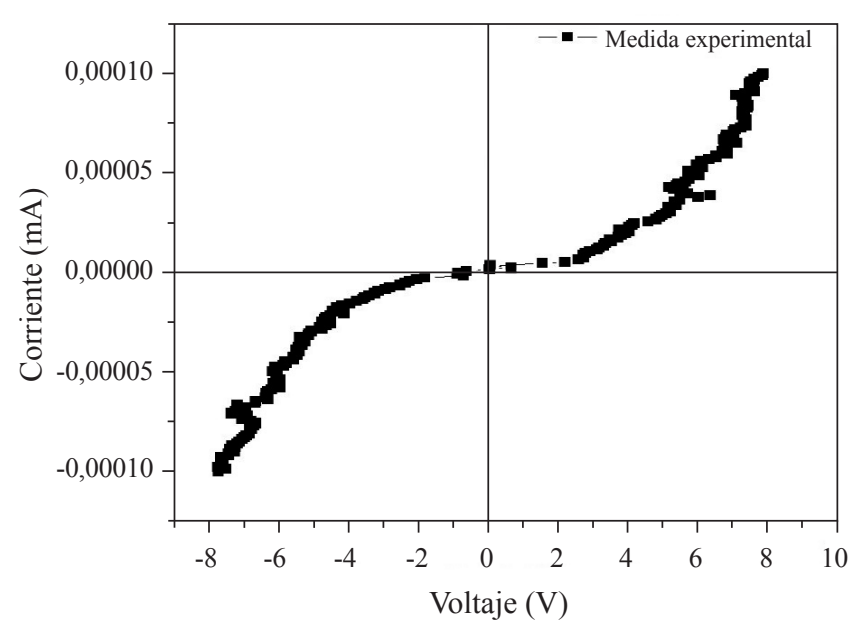

Figura 6. Curvas de intensidad-voltaje en micropuente elaborado con nanoplaquetas obtenidas de Guadua angustifolia Kunth, se puede observar una respuesta eléctrica similar a la de un diodo de punto de contacto.

a la percolación de portadores de carga eléctrica entre las nanoplaquetas, tal como ha sido reportado por He $\boldsymbol{\&}$ Tjong (2013). Además, la aglomeración de las nanoplaquetas podría deberse a los efectos electrostáticos originados por sus formas irregulares, como se puede observar en las micrografías de transmisión de la Figura 5.

Con base en el análisis de curvas I-V en micropuentes de nanoplaquetas, se determinó un valor de conductividad eléctrica de $69 \times 10^{-3} \mathrm{~S} / \mathrm{m}$, el cual concuerda con el orden de magnitud propuesto por Geng, et al. (2009) para el caso de nanoplaquetas de grafito oxidado. Estos resultados sugieren que las nanoplaquetas podrían emplearse en el desarrollo de sensores electrónicos y dispositivos avanzados (Wen-Pin, et al., 2010).

\section{Conclusiones}

Se explica aquí la fabricación y la caracterización de muestras de carbón activado y nanoplaquetas de carbón obtenidas a partir del precursor Guadua angustifolia Kunth para aplicaciones en electrónica. Empleando muestras de carbón activado como electrodo, se propuso un prototipo de dispositivo supercondensador flexible, el cual presentó una capacitancia específica en el $\mathrm{CA}-\mathrm{NaCl}$ y el $\mathrm{CA}-\mathrm{KCl}$ de $111 \mathrm{~F} / \mathrm{g}$ y $79 \mathrm{~F} / \mathrm{g}$, respectivamente, y una capacitancia eléctrica de $7,9 \mathrm{mF}$ en un dispositivo flexible y de dimensiones reducidas. Usando muestras de nanoplaquetas de carbón se logró una configuración de micropuente electrónico con respuestas del tipo del diodo de punto de contacto, y en la caracterización eléctrica en corriente continua se encontraron efectos no lineales atribuidos a la percolación de la corriente eléctrica en el dispositivo. Los resultados sugieren que las muestras de carbón activado y de nanoplaquetas de carbón obtenidas del precursor $G$. angustifolia Kunth podrían utilizarse en el desarrollo de sensores electrónicos y dispositivos avanzados.

\section{Información suplementaria}

Figura 1S. Voltagrama correspondiente a $\mathrm{CA}-\mathrm{KCl}$

\section{Agradecimientos}

Este trabajo recibió el apoyo financiero de la Universidad del Quindío y de Colciencias.

\section{Conflicto de intereses}

Los autores no tienen ningún conflicto de intereses que pueda de alguna manera influir en la transparencia u objetividad en el proceso de revisión por pares y de publicación.

\section{Bibliografía}

Gamby, J., Taberna, P. L., Simon, P., Fauvarque, J. F., Chesneau, M. (2001). Studies and characterisations of various activated carbons used for carbon/carbon supercapacitors. Journal of Power Sources. 101: 109-116. Recuperado de http://www.sciencedirect.com/science/ article/pii/S0378775301007078

Guerrero-González, D.R. (2011). Desarrollo de materiales nanoestructurados basados en óxidos de manganeso con uso potencial en electrodos para dispositivos de almacenamiento de energía (Tesis de maestría). Universidad Nacional de Colombia. Manizales.

Geng, Y., Wang, S. J., Kim, J. K. ( 2009). Preparation of graphite nanoplatelets and graphene sheets. Journal of Colloid and Interface Science. 336: 592-598. Recuperado de http://www. sciencedirect.com/science/article/pii/S0021979709004147

He L. X. \& Tjong S. C. (2013). Zener tunneling in conductive graphite/epoxy composites: Dielectric breakdown aspects. eXPRESS Polymer Letters. 7 (4): 375-382.

Jayalakshmi, M. \& Balasubramanian, K. (2008). Simple capacitors to supercapacitors - An overview. International Journal Electrochemical Science. 3: 1196-1217. Recuperado de http:/www.electrochemsci.org/papers/vol3/3111196.pdf

Lesme J. R. (2013). Pirolisis de biomasa. Experiencias y aplicaciones. Manuscrito en preparación. Centro de Estudios de Eficiencia Energética. Recuperado de http://cengidoc. cengican.org/Portal/SubOtras Areas/Cogeneracion/ Presentaciones/PirolisisBiomasa.pdf

Liu, Y., Zhao, Y., Zhang, Y. (2014). One-step green synthesized fluorescent carbon nanodots from bamboo leaves for copper (II) ion detection. Sensors and Actuators B. 196: 647652. Recuperado de http://www.sciencedirect.com/science/ article/pii/S0925400514002019

Llobet, E. (2013). Gas sensors using carbon nanomaterials: A review. Sensors and Actuators B. 179: 32-45. Recuperado de http://www.sciencedirect.com/science/article/ pii/S0925400512011938

Pandolfo, A. G. \& Hollenkamp, A. F. (2006). Carbon properties and their role in supercapacitors. Journal of power sources. 
157: 11-27. Recuperado de http://www.sciencedirect.com/ science/article/pii/S0378775306003442

Prías Barragán, J. J., Rojas González, C. A., Echeverry Montoya, N. A., Fonthal, G., Ariza-Calderón, H. (2011). Identificación de las variables óptimas para la obtención de carbón activado a partir del Precursor Guadua angustifolia Kunth. Revista de la Academia Colombiana de Ciencias Exactas y de la Tierra. 35 (135): 157-166.

Rozploch, F., Patyk, J., Stankowski, J. (2007). Graphenes bonding forces in graphite. Acta Physica Polonica A. 112 (3): 557-562, Recuperado de http://przyrbwn.icm.edu.pl/ APP/PDF/112/a112z308.pdf
Sangwan, V. K., Southard, A., Moore, T. L., Ballarotto, V. W., Hines, D. R., Fuhrer, M. S., Williams, E. D. (2011). Transfer printing approach to all-carbon nanoelectronics. Microelectronic Engineering. 88: 3150-3154.

Wei, L., \& Yushin, G. (2012). Nanostructured activated carbons from natural precursors for electrical double layer capacitors. Nano Energy. 1: 552-565. Recuperado de http://www. sciencedirect.com/science/article/pii/S2211285512001097

Wen-Pin S., Li-Chi, T., Chian-Wen, L., Ming-Yuan, C., Chienliu, C., Yao-Joe, Y., Kuang-Chao, F. (2010). Flexible temperature sensor array based on a graphitepolydimethylsiloxane composite. Sensors. 10: 3597-3610. 